\title{
The Research of Integrated-Programmable Power Control of Thermal Vacuum Test for Sustainable Energy Development
}

\author{
Yao Feng, Jing Wang, Tao Liao, Dongliang Wua ${ }^{a}$ Xi Zhu, Wanqing An, \\ Bin Shen
}

Beijing Institute of Spacecraft Environment Engineering, Beijing, 100094, China.

aALWAYSLQF@163.com

\begin{abstract}
There hasn't been a quick and easy way to physically connect outdated test instruments to your computer, most engineers need to program and control test instruments and communicate with them from a PC or laptop, It wound wasted a lot of time and effort getting your instruments hooked up, dealing with driver issues and writing code. Thermal vacuum test and thermal balance test are required in the development process of aerospace equipment, the integrated technology of thermal vacuum test and thermal balance test can more reasonably organize the thermal test process and further improve the development efficiency, in order to study the achievable integration of thermal test technology. the internal connection between two kinds of thermal tests was analyzed, and a test method of using one thermal boundary temperature control simulation device to complete two thermal tests in a test was proposed. Based on this, a simulation example of the integration of a program-controlled power supply for a project was used to verify the correctness of the integrated technology, that can simplify outdated test instruments connection, communication, and programming tasks.
\end{abstract}

Keywords: Thermal Vacuum Test, Thermal Balance Test, Integrated-Programmable Power Control.

\section{Introduction}

In order to adapt to the influence of various thermal environments during the entire orbital operation, the correct thermal design must be performed during the development process. Meanwhile, thermal tests are also needed to verify the thermal performance and function of the thermal design and inspection equipment. The thermal tests under the vacuum state of electronic equipment mainly include thermal vacuum tests and thermal balance tests. The thermal vacuum test was performed at the initial stage and the positive stage of the product, and only the thermal balance test was performed at the initial stage. The electronic equipment thermal vacuum test and heat balance test are completely independent two tests. Two completely different thermal boundary simulators and other ancillary equipment are needed[1].

Aerospace products must be properly thermally designed during the development process. Thermal tests are also required to verify thermal performance and functionality of thermal design and inspection equipment.

Thermal boundary simulation of thermal vacuum test of electronic equipment usually uses infrared cage. By controlling the temperature of the infrared cage, the high and low temperature requirements of the equipment are achieved. The thermal vacuum test requires several high and low temperature cycles to be completed as specified.

The basic steps of the thermal vacuum test are as follows: The temperature control point starts at the ambient temperature and rises to the specified temperature and is stable. After the specified time, the corresponding performance and function tests are performed. Then temperature control point temperature down to the specified temperature and is stable after power off, stay for the specified time, and perform the appropriate performance and functional testing, after completion of the temperature rise, the temperature control point when the temperature reaches the ambient temperature performs a temperature cycle[2].

Thermal boundaries of thermal equilibrium tests typically use aluminum plates to simulate the installed thermal environment on the satellite. The electronic device is mounted on a base plate coated with a thermal conductive material such as thermal grease, and the other pods simulate a radiant heat 
boundary, and a high emissive coating is usually applied to the inner surface of the electronic device. The basic practice of the thermal balance test is as follows: The control thermal boundary is the specified low temperature limit or high temperature limit. After the temperature stabilizing, the electronic device continuously being tested until the specified time and then power off.

Combined with the above analysis, the thermal boundary simulation of both thermal tests in this paper uses infrared cage devices[3].

\section{Experimental Setup}

From the initial stage of development, each spacecraft began to study its thermal mathematical model in outer space. Regardless of the use of the integral form of the ordinary differential equation, or the use of the definite solution of partial differential equations, the spacecraft temperature field can be obtained using equivalent boundary conditions and initial conditions. In general, depending on the choice of thermal properties parameters, especially the contact thermal resistance at the junction and the contact surface, and the selection of the absorption rate and emissive factor, those needed to be experimentally verified. Moreover, for a considerable period of time, the thermal balance test is the basis for evaluation and improvement of the thermal control subsystem. This requires the thermal control system to have accurate heating capacity. For the thermal control system, the heating loop of several tens or even hundreds of loops must be performed[4,5]. A series of temperature-controlled, program-controlled power supplies are required for control. Therefore, an integrated thermal test technology based on programmable power supplies is particularly important.

The field bus technology that emerged in the 1990s has brought great influence on the development of industrial measurement and control systems. The huge economic benefits it generates have caused the European and American large-scale control equipment production groups to launch fierce competition around the international standards of field bus in the past decade. The International Electrotechnical Commission (IEC)[6] passed the IEC61158 international standard in January 2000. The standard includes eight types of field bus standards: FF-H1, Control Net, P-NET, FF-HSE, Swift Net, Wor1dFIP and Inter-bus. Each type of field bus uses completely different communication protocols and it is difficult to achieve mutual compatibility of these buses. The rapid development of Ethernet technology has laid a solid foundation for the industry's field observation and control[7,8], and Ethernet has become an inevitable trend of development.

In this paper, in order to solve the problem of uncontrollable brought about by the interface of old devices without LANs, this is also the need for the sustainable development of instruments and equipment, the program-controlled power converter based on RS232 and RS485 interface are developed to convert program-controlled power to Ethernet, and the network-based intelligent thermal control of program-controlled power supply is realized. The integrated thermal test technology of program-controlled power supply can be realized with the upper computer network management software.

The RS485-LAN converter system consists of the host computer network control software and protocol converter, as shown in Fig1.

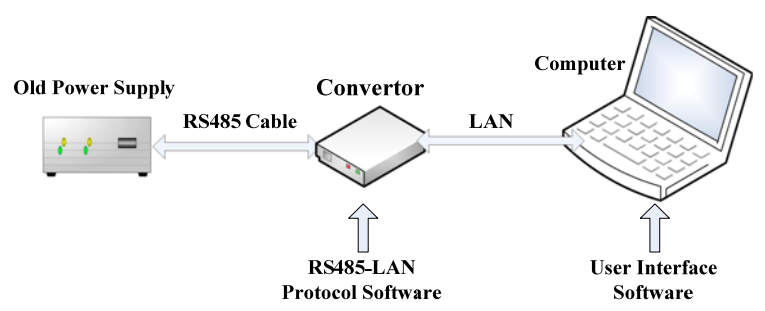

Fig 1.RS485-LAN converter system composition

The RS485-LAN converter interface info. is listed in Table 1. RS485-LAN converter Real photos are shown in Fig 2, block diagram and schematic is shown in Fig 3. 
Table 1.RS485-LAN converter interface info.

\begin{tabular}{|c|c|c|}
\hline$\#$ & Interface name & Interface info. \\
\hline 1 & Power Supply & AC220V plugin \\
\hline 2 & Communication interface down to old power equipment & 6 pin RJ11 \\
\hline 3 & Communication interface up to computer & LAN \\
\hline 4 & Debug interface & USB-A \\
\hline 5 & Power and communication indicator display & 0.96 inch yellow-blue OLED \\
\hline
\end{tabular}

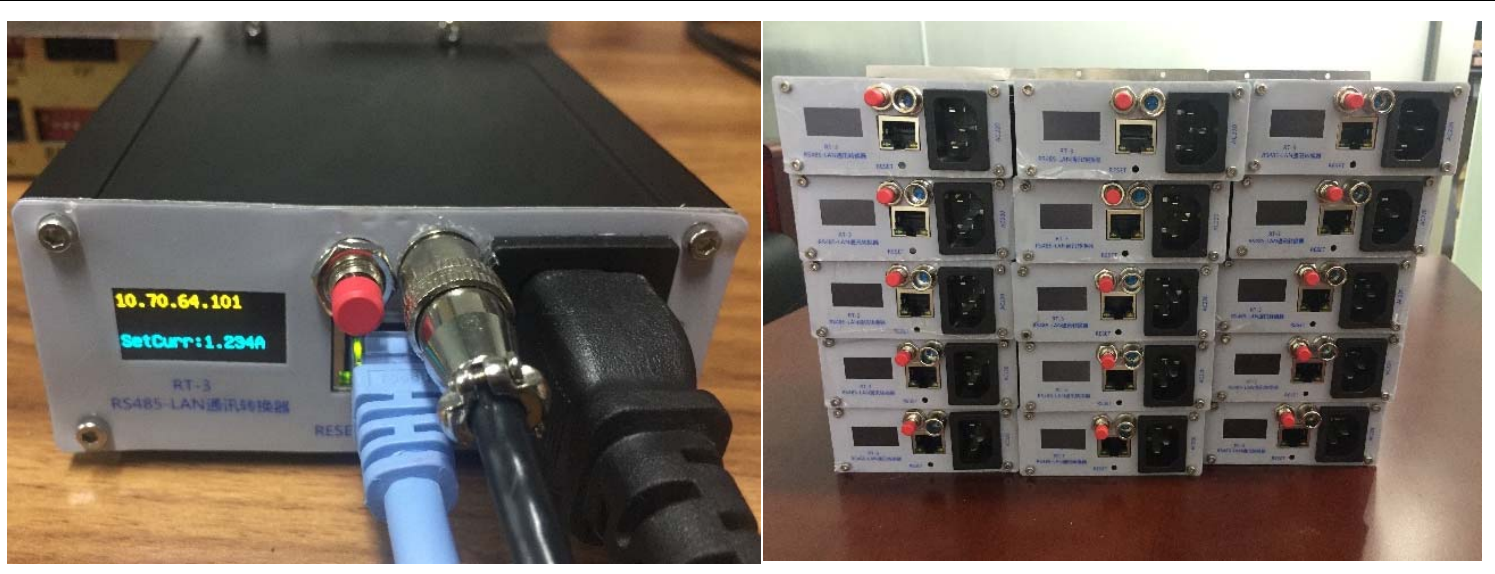

Fig 2.RS485-LAN converter Real photos

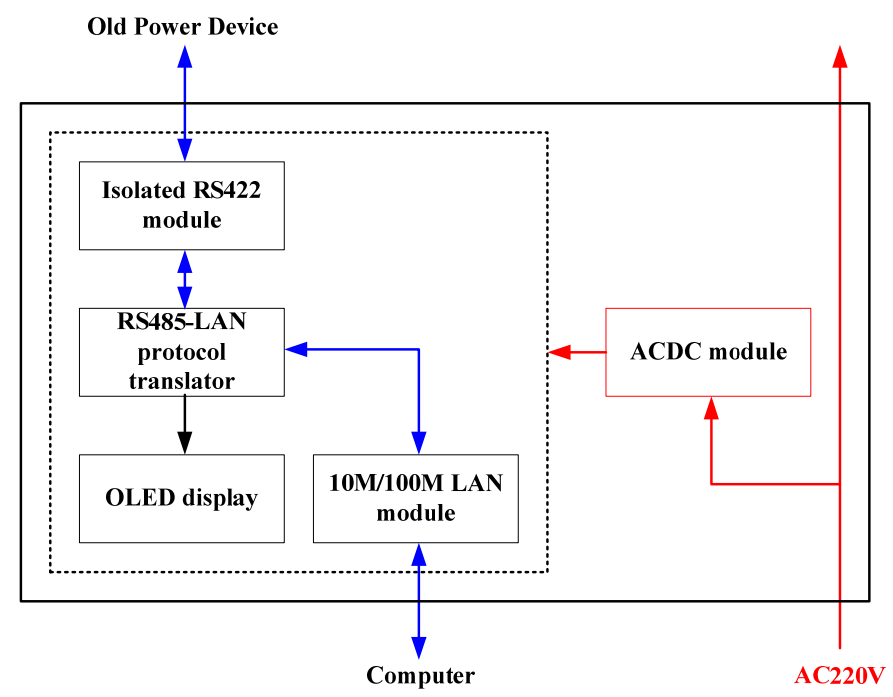

Fig 3.Hardware design block diagram

\section{Experimental Result}

The software for the upper computer is on the platform of the $\mathrm{x} 86$ computer Windows XP system, with LabVIEW (Laboratory Virtual Instrument Engineering Workbench) develop environment, based on standard MFC or WIN32 function sets, dialog model, those makes user interface program is easy to use, as shown in Fig 4.

Parameter setting page, enter the server IP, and use this IP to try the TCP connection; set the power model, when connected to the RS485-LAN converter on the server, notify the RS485-LAN converter to use the model of the radio command set.

Use Socket to create a TCP client and connect to the server. Only the successful connection to the server can control power and modify the server IP. 


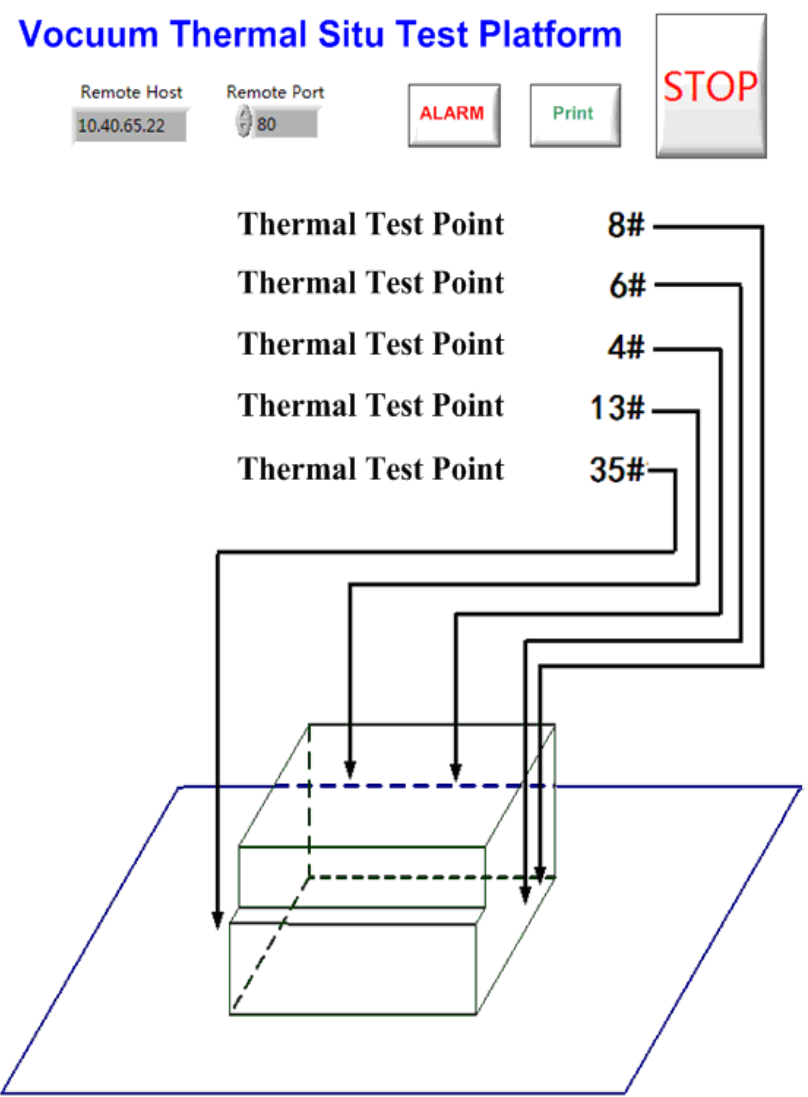

Fig 4.Thermal test PC software interface

Here we figure two test point temperature, thermal test point $4 \#$ and $8 \#$ temperature spot curve as shown in Fig 5 and Fig 6, separately.

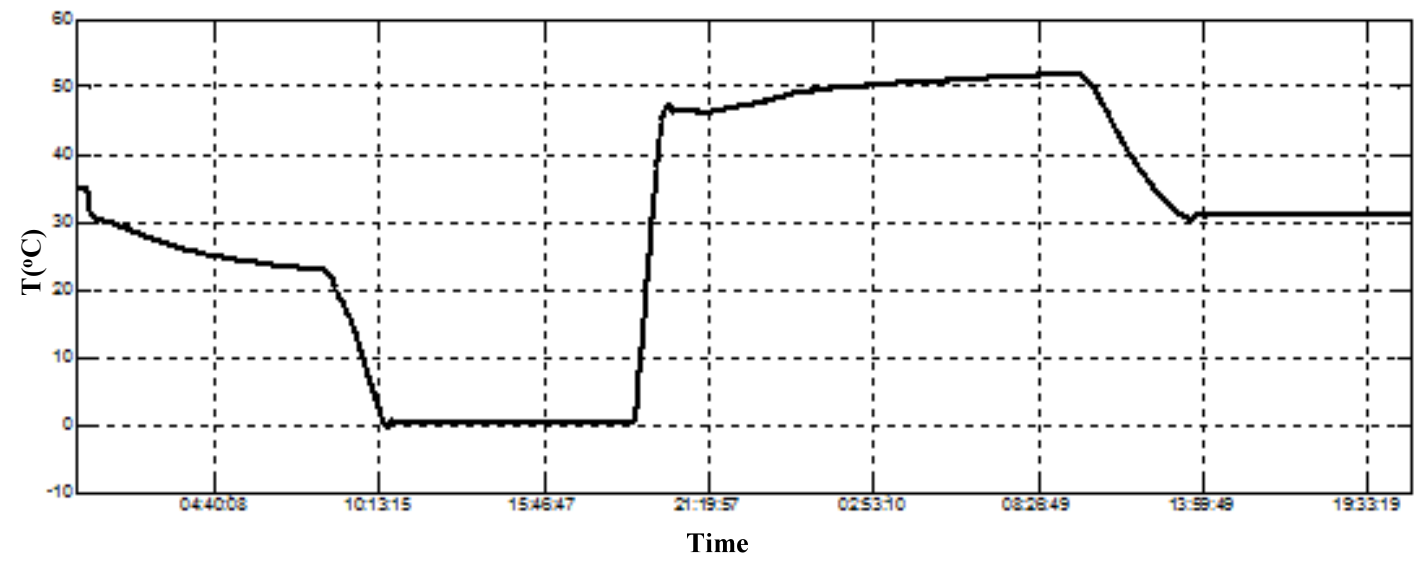

Fig 5.Thermal test point $4 \#$ temperature spot curve 


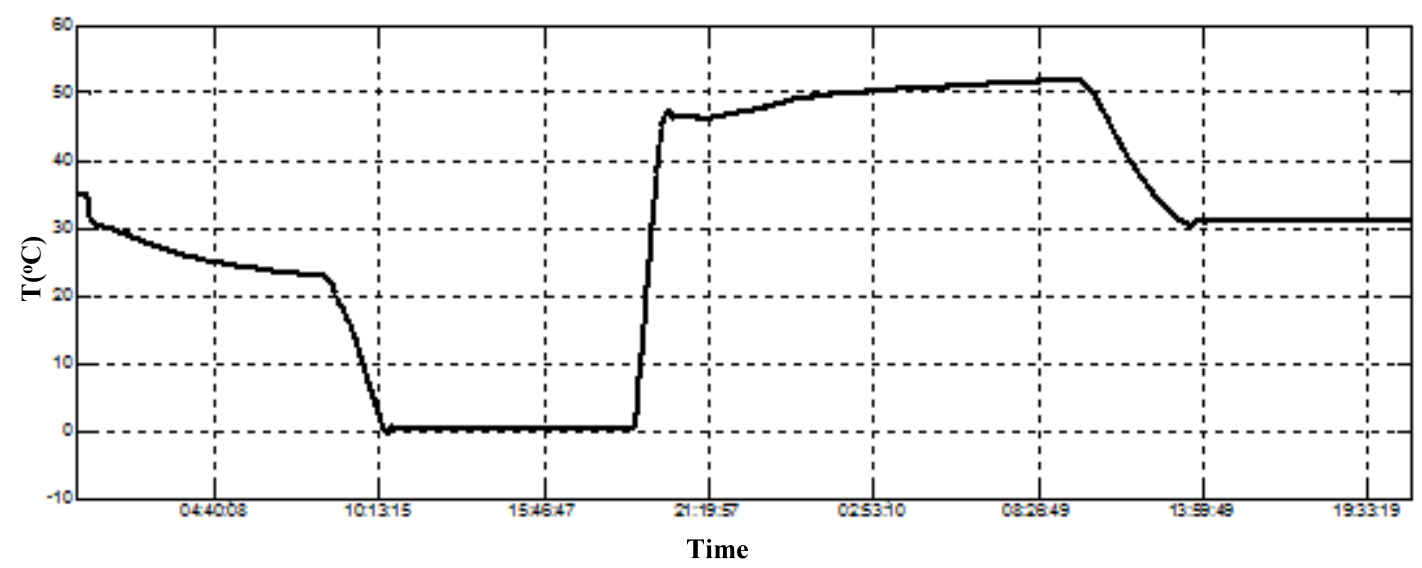

Fig 6.Thermal test point $8 \#$ temperature spot curve

\section{Summary}

Until recently, there hasn't been a quick and easy way to physically connect outdated test instruments to your computer, much less an easy way to get your PC and test equipment to communicate smoothly with each other. The significance of the integration technology of thermal vacuum test and thermal balance test of electronic equipment is informed. The integration technology of thermal test can provide test data for the subsequent test, and further judge the reliable and safe conduct of the test. In addition, this paper is to introduce some of the hardware options available that can simplify outdated test instruments connection, communication, and programming tasks.

\section{References}

[1]. 'Auria, M. D., Lovaglio, T., Rita, A., Cetera, P., Romani, A., \& Hiziroglu, S., et al. (2018). Integrate measurements allow the surface characterization of thermo-vacuum treated alder differentially coated. Measurement, 114, 372.

[2]. Kudlac, Maureen, H. Weaver, and M. Cmar. "NASA Plum Brook's B-2 test facility-Thermal vacuum and propellant test facility." American Institute of Physics, 2012:1253-1260.

[3]. Swamy Kidambi, Rajamannar, et al. "Design and performance of vacuum system for high heat flux test facility." Journal of Physics Conference Series Journal of Physics Conference Series, 2017:012024.

[4]. Hasan, M. A., Rashmi, S., Esther, A. C. M., Sherikar, B. N., Sridhara, N., \& Dey, A. (2018). Evaluations of silica aerogel-based flexible blanket as passive thermal control element for spacecraft applications. Journal of Materials Engineering \& Performance, 27(3), 1265-1273.

[5]. Gandhi, J., \& Pathak, A. V. (2012). Performance evaluation of thermal interface material for space applications. Applied Mechanics \& Materials, 110-116, 135-141.

[6]. Information on: https://en.wikipedia.org/wiki/International_Electrotechnical_Commission.

[7]. Liu, C., Fan, X. L., Wang, J., Xue, L., \& Wang, Y. Y. (2018). The design and verification of the temperature controllable canister in thermal vacuum environment. Young Scientists Forum (pp.111).

[8]. Chin, K. C., Green, N. W., \& Brandon, E. J. (2018). Evaluation of supercapacitors for space applications under thermal vacuum conditions. Journal of Power Sources, 379, 155-159. 